\title{
TRANSFERENCE FOR RADIAL MULTIPLIERS AND DIMENSION FREE ESTIMATES
}

\author{
P. AUSCHER AND M. J. CARRO
}

\begin{abstract}
For a large class of radial multipliers on $L^{p}\left(\mathbf{R}^{\mathbf{n}}\right)$, we obtain bounds that do not depend on the dimension $n$. These estimates apply to well-known multiplier operators and also give another proof of the boundedness of the Hardy-Littlewood maximal function over Euclidean balls on $L^{P}\left(\mathbf{R}^{\mathbf{n}}\right), p \geq$ 2 , with constant independent of the dimension. The proof is based on the corresponding result for the Riesz transforms and the method of rotations.
\end{abstract}

\section{INTRODUCTION}

The aim of this paper is to give a unified approach to obtain the boundedness of certain radial multipliers and maximal operators on $L^{p}\left(\mathbf{R}^{\mathbf{n}}\right)$ such as Bochner-Riesz means or the Hardy-Littlewood maximal operator. We do this by employing transference and variations of the method of rotations. We obtain precise estimates for the norms of these operators acting on $L^{p}\left(\mathbf{R}^{\mathbf{n}}\right)$ as a function of $n$.

These types of studies were initiated by E. Stein and J. O. Strömberg in [SS]. In this paper they proved that the Hardy-Littlewood maximal operator over Euclidean balls, $M$, satisfies the inequality $\|M f\|_{p} \leq C(p)\|f\|_{p}$ for $f \in$ $L^{p}\left(\mathbf{R}^{\mathbf{n}}\right)$ where $C(p)<+\infty \quad(p>1)$ is bounded as the dimension $n$ increases. One of our goals was to provide a proof of this result that gives for $C(p)$ a numerical value bounded independently of $n$. As a consequence of our study we obtain a partial answer.

Theorem A. If $n \geq 2$ and $p \geq 2$ then $\|M f\|_{p} \leq(2+\sqrt{2})^{2 / p}\|f\|_{p}$.

The method of the proof relies on the observation that the characteristic function of the ball $\chi_{B}$ has a Fourier transform that can be seen as a linear combination of radial multipliers in a certain class. The latter are of the form

$$
M_{\varphi}^{m, \alpha}(\xi)=c_{m, n, \alpha} \int_{-\infty}^{+\infty} \varphi(t) \frac{J_{m+\alpha+(n-2) / 2}(2 \pi t|\xi|)}{(t|\xi|)^{\alpha+(n-2) / 2}} d t, \quad m \in \mathbf{Z}^{+}, \alpha \geq 0
$$

Received by the editors August 16, 1990 and, in revised form, July 22, 1991 and February 13, 1992.

1980 Mathematics Subject Classification (1985 Revision). Primary 42B15, 42B25.

Key words and phrases. Radial multipliers, transference.

The second author was on leave from Universitat de Barcelona, Spain, and partially supported by DGICYT (PS87-0027). 
where $\varphi$ is a function on $\mathbf{R}$, the function $J_{\lambda}(z) / z^{\lambda}$ is defined (see [WW, p. 358]) for all complex values $z$ and $\lambda$ by the power series

$$
\frac{J_{\lambda}(z)}{z^{\lambda}}=\sum_{n=0}^{\infty} \frac{(-1)^{n} z^{2 n}}{n ! 2^{2 n+\lambda} \Gamma(\lambda+n+1)}
$$

and $c_{m, n, \alpha}$ is a "natural" constant which will be defined in the course of the paper. For example, the multipliers $\hat{\chi}_{B},|\xi|^{m}\left(1-|\xi|^{2}\right)_{+}^{\delta},|\xi|^{i \gamma}, \gamma \in \mathbf{R}$, can be obtained from these multipliers with suitable choices of $m, \alpha$ and $\varphi$.

The Riesz transforms of higher order are highly related to the above family of multipliers and we make use of the following inequality due to Stein [S1]

\section{Theorem B.}

$$
\left\|\left(\sum_{j}\left|R_{j}^{m} f\right|^{2}\right)^{1 / 2}\right\|_{p} \leq K(m, p)\|f\|_{p}
$$

for $1<p<+\infty, m=1,2, \ldots$.

Here $R_{j}^{m}, j=1,2, \ldots, d(m, n)$ (see $\S 1$ ) denote the family of higher Riesz transforms of order $m$ and $K(m, p)$ is independent of the dimension $n$. By different arguments, this inequality has also been obtained by Duoandikoetxea and Rubio de Francia [DR] and Pisier [P]. Extending the ideas in [DR] and using a variant of the method of rotations, we can show

Theorem C.

$$
\left\|\check{M}_{\varphi}^{m, \alpha} * f\right\|_{p} \leq K\left(m, p^{\prime}\right) N_{p}(\varphi)\|f\|_{p}
$$

for all $1<p<\infty, m=1,2, \ldots$, and $\alpha \geq 0$.

Here $p^{\prime}=p /(p-1)$ and $N_{p}(\varphi)$ is the norm of the convolution operator on $L^{p}(\mathbf{R})$ with kernel $\varphi(t)$. This provides estimates independent of the dimension for this class of radial multipliers and we recover results already observed at the end of [S1].

To obtain maximal estimates we first notice that if $\varphi_{r}(t)=\varphi(t / r) / r$ for $r>0$, then $M_{\varphi_{r}}^{m}(\xi)=M_{\varphi}^{m}(r \xi)$. This suggests a result of transference type for maximal inequalities.

Theorem D. If $\varphi \geq 0$ and $\sup _{r>0}\left|\varphi_{r} * g\right|$ satisfies a maximal inequality in $L^{p}(\mathbf{R})$ for all $1<p \leq \infty$ with bound $A_{p}$ then, for all $1<s<\min (2, p) \leq p<\infty$

$$
\left\|\sup _{r>0}\left|\check{M}_{\varphi_{r}}^{m} * f\right|\right\|_{L^{p}\left(\mathbf{R}^{\mathbf{n}}\right)} \leq C(m, p, s) n^{m / 2} A_{p / s}^{1 / s}\|f\|_{L^{p}\left(\mathbf{R}^{\mathbf{n}}\right)} .
$$

A shortcoming of this result is the dependence on the dimension of the bound in the above inequality. For $p=2$, another approach is available.

Theorem E. Let $m \geq 1, n \geq 2$ and assume $\operatorname{supp} \varphi \subset[0,+\infty]$, then

$$
\left\|\sup _{r>0}\left|\check{M}_{\varphi_{r}}^{m} * f\right|\right\|_{L^{2}\left(\mathbf{R}^{\mathbf{n}}\right)} \leq\left(\int_{0}^{\infty} t|\varphi(t)|^{2} d t\right)^{1 / 2} m^{-1 / 2}\|f\|_{L^{2}\left(\mathbf{R}^{\mathbf{n}}\right)} .
$$

This estimate can be shown to be sharp. As a consequence of Theorem $\mathrm{E}$ we obtain Theorem A and, for example, another proof of the boundedness of the maximal Bochner-Riesz means of positive order on $L^{2}\left(\mathbf{R}^{\mathbf{n}}\right)$. 
The paper is organized as follows. In the first section, we prove Theorem $\mathrm{C}$. Maximal Theorems D and E are proved in $\S 2$. They are used to prove Theorem A and to give a new argument for the $L^{2}$ boundedness of the maximal BochnerRiesz means of positive order. In $\S 3$, we extend Theorem A to a more general class of maximal operators and conclude the paper with some remarks.

We are indebted to Professor Guido Weiss for a series of lectures he gave on the transference methods that he developed with R. Coifman [CW], while both of us were in the Department of Mathematics of Washington University in St. Louis. He introduced both of us to these topics and led us to the study of these problems. We thank him also for the numerous comments he made on our work.

\section{RADIAL MULTIPLIERS OBTAINED BY TRANSFERENCE}

Let us explain in a few words the proof given by G. Pisier of the boundedness of the Riesz transforms on $L^{p}\left(\mathbf{R}^{\mathbf{n}}\right)$. This is a natural introduction to our study.

For $f \in \mathscr{S}\left(\mathbf{R}^{\mathbf{n}}\right)$ and $y \in \mathbf{R}^{\mathbf{n}}$, consider the Hilbert transform on $\mathbf{R}^{\mathbf{n}}$ acting in the direction $y$ :

$$
H^{y} f(x)=\text { p.v. } \int_{-\infty}^{+\infty} \frac{1}{t} f(x-t y) d t
$$

A well-known transference argument (see [CW]) shows that for all $y \in \mathbf{R}^{\mathbf{n}}$ :

$$
\left\|H^{y} f\right\|_{L^{p}\left(\mathbf{R}^{\mathbf{n}}\right)} \leq H_{p}\|f\|_{L^{p}\left(\mathbf{R}^{\mathbf{n}}\right)}, \quad 1<p<\infty
$$

where $H_{p}$ is the norm of the 1-dimensional Hilbert transform. This estimate is uniform in $y$. Now, equip $\mathbf{R}^{\mathbf{n}}$ with the Gaussian probability measure $d \gamma_{n}(y)=$ $(2 \pi)^{-n / 2} e^{-|y|^{2} / 2} d y$ and introduce the orthogonal projection $Q$ of $L^{2}\left(\mathbf{R}^{\mathbf{n}}, \gamma_{n}\right)$ onto the space of functions of the form $\sum_{j=1}^{n} a_{j} y_{j}$ in which the coordinate functions $\left\{y_{j}\right\}_{j=1, \ldots, n}$ form an orthonormal basis. Then, for a.e. $x \in \mathbf{R}^{\mathbf{n}}$, Pisier obtained the formula:

$$
Q\left[H^{\cdot} f(x)\right](y)=\sqrt{\frac{2}{\pi}} \sum_{j=1}^{n} R_{j} f(x) y_{j}
$$

where $R_{j}$ is the $j$ th Riesz transform defined by $\left(\widehat{R_{j} f}\right)(\xi)=-i\left(\xi_{j} /|\xi|\right) \hat{f}(\xi)$. The equalities

(**) $\quad\left\|\sum_{j=1}^{n} a_{j} y_{j}\right\|_{L^{p}\left(\mathbf{R}^{\mathbf{n}}, \gamma_{n}\right)}=\gamma(p)\left\|\sum_{j=1}^{n} a_{j} y_{j}\right\|_{L^{2}\left(\mathbf{R}^{\mathbf{n}}, \gamma_{n}\right)}=\gamma(p)\left(\sum_{j=1}^{n}\left|a_{j}\right|^{2}\right)^{1 / 2}$,

$1 \leq p<\infty$, where $\gamma(p)=\left(\int_{-\infty}^{+\infty}|t|^{p} e^{-t^{2} / 2}(2 \pi)^{-1 / 2} d t\right)^{1 / p}$, imply that

$$
\|Q g\|_{L^{p}\left(\mathbf{R}^{\mathbf{n}}, \gamma_{n}\right)} \leq \max \left(\gamma(p), \gamma\left(p^{\prime}\right)\right)\|g\|_{L^{p}\left(\mathbf{R}^{\mathbf{n}}, \gamma_{n}\right)},
$$

for all $g \in L^{p}\left(\mathbf{R}^{\mathbf{n}}, \gamma_{n}\right) \cap L^{2}\left(\mathbf{R}^{\mathbf{n}}, \gamma_{n}\right)$ and $1<p<\infty$. 
From $(*),(* *)$ and this last inequality, Pisier obtains the result as follows:

$$
\begin{aligned}
\left\|\left(\sum_{j=1}^{n}\left|R_{j} f(x)\right|^{2}\right)^{1 / 2}\right\|_{L^{p}\left(\mathbf{R}^{\mathbf{n}}\right)} & =\frac{1}{\gamma(p)}\|\| \sum_{j=1}^{n} R_{j} f(x) y_{j}\left\|_{L^{p}\left(\mathbf{R}^{\mathbf{n}}, \gamma_{n}\right)}\right\|_{L^{p}\left(\mathbf{R}^{\mathbf{n}}\right)} \\
& \leq \frac{1}{\gamma(p)} \sqrt{\frac{\pi}{2}}\|\| Q[H \cdot f(x)](y)\left\|_{L^{p}\left(\mathbf{R}^{\mathbf{n}}, \gamma_{n}\right)}\right\|_{L^{p}\left(\mathbf{R}^{\mathbf{n}}\right)} \\
& \leq \max \left(1, \frac{\gamma\left(p^{\prime}\right)}{\gamma(p)}\right) \sqrt{\frac{\pi}{2}}\|\| H^{y} f(x)\left\|_{L^{p}\left(\mathbf{R}^{\mathbf{n}}, \gamma_{n}\right)}\right\|_{L^{p}\left(\mathbf{R}^{\mathbf{n}}\right)} \\
& \leq H_{p} \max \left(1, \frac{\gamma\left(p^{\prime}\right)}{\gamma(p)}\right) \sqrt{\frac{\pi}{2}}\|f\|_{L^{p}\left(\mathbf{R}^{\mathbf{n}}\right)} .
\end{aligned}
$$

The last inequality is obtained, after a change in the order of integration, from the transference result for the Hilbert transform in the $y$ direction and from the fact that $\left(\mathbf{R}^{\mathbf{n}}, \gamma_{n}\right)$ is a probability space.

As we can see, the two main tools used in this outline of the proof are the application of the special projection $Q$ followed by that of an operator whose boundedness can be obtained by transference. Our first theorem will make use of these ideas.

We feel that the computations made in $\mathbf{R}^{\mathbf{n}}$ equipped with the Gaussian measure are somewhat artificial since the "natural" setting for the variable $y$ is the unit sphere $\Sigma_{n-1}$ endowed with the rotational invariant measure $d y^{\prime}$ normalized so that $\int_{\Sigma_{n-1}} d y^{\prime}=1$. It turns out that a formula similar to $(*)$ can be obtained in this setting. A heuristic argument which might explain the method of Pisier is that $\left(\mathbf{R}^{\mathbf{n}}, \gamma_{n}\right)$ can be seen as an asymptotic version of $\left(\Sigma_{n-1}, d y^{\prime}\right)$. (For a justification of this see Vilenkin, Chapter XI of [V].) Moreover, by making this use of the sphere, as we explain later, we are closer to the method developed by Duoandikoetxea and Rubio de Francia.

Let us now present our first result. For $n \geq 2$ and $m \geq 0$, let $H^{m}$ be the space of spherical harmonics (restricted to $\Sigma_{n-1}$ ) of degree $m$ and $d(m, n)=$ $\operatorname{dim} H^{m}$. We have (see [SW, Chapter IV]) $d(0, n)=1, d(1, n)=n$ and

$$
d(m, n)=\frac{(m+n-3) !(2 m+n-2)}{(n-2) ! m !} .
$$

Let $J_{\alpha}$ denote the Bessel function of order $\alpha$ and $b_{n-2}=\pi^{n / 2-1} / \Gamma(n / 2)$ the volume of the unit ball in $\mathbf{R}^{\mathbf{n}-2}$. We make the convention that $b_{0}=1$ (which is consistent with the formula when $n=2$ ).

Theorem 1. Let $1<p<\infty$ and $\varphi(t)$ be a convolution kernel acting on $L^{p}(\mathbf{R})$ with operator norm $N_{p}(\varphi)$. Then, for $n \geq 2, m \geq 1$, the function

$$
M_{\varphi}^{m}(\xi)=\frac{\sqrt{d(m, n)}}{b_{n-2}} \int_{-\infty}^{+\infty} \varphi(t) \frac{J_{m+(n-2) / 2}(2 \pi t|\xi|)}{(t|\xi|)^{(n-2) / 2}} d t
$$

defines a multiplier of $L^{p}\left(\mathbf{R}^{\mathbf{n}}\right)$ such that

$$
\left\|\check{M}_{\varphi}^{m} * f\right\|_{L^{p}\left(\mathbf{R}^{\mathbf{n}}\right)} \leq C(m, p) N_{p}(\varphi)\|f\|_{L^{p}\left(\mathbf{R}^{\mathbf{n}}\right)},
$$

with $C(m, p)$ independent of the dimension. 
As we shall see, if we take $\varphi(t)=1 / t$ and $m$ odd, we get

$$
M_{\varphi}^{m}(\xi)=\frac{\sqrt{d(m, n)}}{b_{n-2}} \int_{-\infty}^{+\infty} \frac{1}{t} \frac{J_{m+(n-2) / 2}(2 \pi t|\xi|)}{(t|\xi|)^{(n-2) / 2}} d t
$$

if $\varphi(t)=1 /|t|^{1+i \gamma}$ for $\gamma \neq 0$ and $m$ even, then

$$
M_{\varphi}^{m}(\xi)=\sqrt{d(m, n)} \pi^{i \gamma} \frac{\Gamma((m-i \gamma) / 2) \Gamma(n / 2)}{\Gamma((n+m+i \gamma) / 2)}|\xi|^{i \gamma},
$$

and, if

$$
\varphi(t)=\frac{2 \pi \Gamma(\beta)}{\pi^{\beta-1}} \frac{b_{n-2}}{\sqrt{d(m, n)}} \frac{J_{m+\beta+(n-2) / 2}(2 \pi t)}{t^{\beta-n / 2}} \chi_{[0,+\infty)}(t)
$$

we get $M_{\varphi}^{m}(\xi)=|\xi|^{m}\left(1-|\xi|^{2}\right)_{+}^{\beta-1}$.

Remarks. (1) For $m=0,1 \leq p \leq \infty$, and $\operatorname{supp} \varphi \subset[0,+\infty)$ the result is also true. However, as we shall see, this is just the method of rotations expressed on the Fourier side. Indeed, $M_{\varphi}^{0}$ is the Fourier transform of the radial kernel $K(x)$ given by $\omega_{n-1}|x|^{n-1} K(x)=\varphi(|x|)$ where $\omega_{n-1}=2 \pi b_{n-2}$ is the surface area of the unit sphere $\Sigma_{n-1}$ in $\mathbf{R}^{\mathbf{n}}$. This explains in part the normalization of the multipliers $M_{\varphi}^{m}$.

(2) For fixed $m, d(m, n)=O\left[n^{m}\right]$ and $b_{n-2}=O\left[(2 \pi e / n)^{(n-1) / 2}\right]$ as $n$ tends to $+\infty$ (this is easily obtained from Stirling's formula). Let us see why the normalization in (1.1) is necessary and best possible. Taking $\varphi(t)=\delta_{1}(t)$ (Dirac mass at $t=1$ ) and $p=2$, the bound of the multipliers $M_{\varphi}^{m}$ on $L^{2}\left(\mathbf{R}^{\mathbf{n}}\right)$ is

$$
\sup _{\xi \in \mathbf{R}^{\mathbf{n}}} \frac{\sqrt{d(m, n)}}{b_{n-2}}\left|\frac{J_{m+(n-2) / 2}(2 \pi|\xi|)}{|\xi|^{(n-2) / 2}}\right|,
$$

and can be compared to $C(m, 2)$ in (1.2). For fixed $m$, using the power series expansion for $J_{\nu}$ (Watson [W, p. 40]), the above supremum can be shown to be bounded from below by a nonnegative constant $C(m)$ for $n$ large enough. Another reason that makes this normalization necessary relies on the relations between these multipliers and the Riesz transforms that we will establish later.

(3) In our arguments, we consider $\varphi \in L^{1}(\mathbf{R})$ with compact support. The general $\varphi$ can be approximated by a sequence $\left\{\varphi_{n}\right\}$ of such kernels so that $\varphi_{n} \rightarrow \varphi$ in the distributional sense and such that $N_{p}\left(\varphi_{n}\right) \leq N_{p}(\varphi)$. The desired estimate with $\varphi$ then follows.

Proof. If $x \in \mathbf{R}^{\mathbf{n}}, y^{\prime} \in \Sigma_{n-1}$ and $f \in \mathscr{S}\left(\mathbf{R}^{\mathbf{n}}\right)$ define $F_{\varphi}\left(x, y^{\prime}\right)$ to be the expression

$$
\int_{-\infty}^{+\infty} \varphi(t) f\left(x-t y^{\prime}\right) d t
$$

For $y^{\prime}$ fixed, $R_{t}^{y^{\prime}} f(x)=f\left(x-t y^{\prime}\right)$ defines a unitary representation of $\mathbf{R}$ acting on $L^{p}\left(\mathbf{R}^{\mathbf{n}}\right)$. By transference (see [CW, p. 9]) we then have

$$
\left\|F_{\varphi}\left(\cdot, y^{\prime}\right)\right\|_{L^{p}\left(\mathbf{R}^{\mathrm{n}}\right)} \leq N_{p}(\varphi)\|f\|_{L^{p}\left(\mathbf{R}^{\mathbf{n}}\right)} \cdot
$$

We emphasize the fact that this estimate is uniform in $y^{\prime}$. 
For example, if we take $\varphi(t)=$ p.v. $\frac{1}{t}$ then $F_{\varphi}\left(x, y^{\prime}\right)=H^{y^{\prime}} f(x)$ the Hilbert transform in the $y^{\prime}$ direction previously defined. Also, if $\varphi(t)=n t^{n-1} \chi_{[0,1]}(t)$ then

$$
\frac{1}{b_{n}} \chi_{B(0,1)} * f(x)=\int_{\Sigma_{n-1}} F_{\varphi}\left(x, y^{\prime}\right) d y^{\prime} .
$$

Let $Q^{m}: L^{2}\left(\Sigma_{n-1}, d y^{\prime}\right) \rightarrow H^{m}$ be the orthogonal projection onto $H^{m}$. Choose an orthonormal basis $\left\{Y_{j}^{m}\right\}_{j=1}^{d(m, n)}$ of $H^{m}$ (of real-valued spherical harmonics) normalized so that

$$
\left\|Y_{j}^{m}\right\|_{L^{2}\left(\Sigma_{n-1}, d y^{\prime}\right)}=\frac{1}{\sqrt{d(m, n)}} .
$$

As in [DR], we introduce the "higher Riesz transforms of order $m$ " by defining their action in the Fourier transform side:

$$
\left(\widehat{R_{j}^{m}} f\right)(\xi)=i^{-m} Y_{j}^{m}\left(\xi^{\prime}\right) \hat{f}(\xi),
$$

$j=1, \ldots, d(m, n)$, where $\xi^{\prime}=\xi|\xi|^{-1}, \xi \neq 0$. The normalization of these higher Riesz transforms is the "natural one" when one considers the "higher Riesz systems" (see [CZ]). Note that for $m=1$, the most natural choice is $Y_{j}^{1}\left(\xi^{\prime}\right)=\xi_{j}^{\prime}$ for which we have $\left\|Y_{j}^{1}\right\|_{L^{2}\left(\Sigma_{n-1}, d y^{\prime}\right)}=n^{-1 / 2}=d(1, n)^{-1 / 2}$. Then, $\left(\widehat{R_{j}^{1} f}\right)(\xi)=i^{-1} \xi_{j}^{\prime} \hat{f}(\xi)$ are the classical M. Riesz transforms. For convenience, when $m=0$, since $H^{0}$ is the space of constant functions, we will take $Y_{j}^{0}\left(\xi^{\prime}\right)=$ 1 and, thus, the "associated Riesz transform" is the identity operator.

We have the following identity:

Proposition 2. Let $M_{\varphi}^{m}(\xi)$ be defined by (1.1) and $f \in \mathscr{S}\left(\mathbf{R}^{\mathbf{n}}\right)$. Then, for a.e. $x \in \mathbf{R}^{\mathbf{n}}$,

$$
Q^{m}\left[F_{\varphi}(x, \cdot)\right]\left(y^{\prime}\right)=\sqrt{d(m, n)} \sum_{j=1}^{d(m, n)} R_{j}^{m}\left(\check{M}_{\varphi}^{m} * f\right)(x) Y_{j}^{m}\left(y^{\prime}\right) .
$$

Proof. By definition

$$
Q^{m}\left[F_{\varphi}(x, \cdot)\right]\left(y^{\prime}\right)=d(m, n) \sum_{j=1}^{d(m, n)} \int_{\Sigma_{n-1}} F_{\varphi}\left(x, z^{\prime}\right) Y_{j}^{m}\left(z^{\prime}\right) d z^{\prime} Y_{j}^{m}\left(y^{\prime}\right) .
$$

Taking the Fourier transform in the variable $x$ (justified by an appropriate use of Fubini's Theorem):

$(*)$

$$
\begin{aligned}
& Q^{m}\left[F_{\varphi}(\hat{\xi}, \cdot)\right]\left(y^{\prime}\right) \\
& \quad=d(m, n) \sum_{j=1}^{d(m, n)}\left(\int_{-\infty}^{+\infty} \varphi(t) \int_{\Sigma_{n-1}} e^{-2 \pi i t z^{\prime} \cdot \xi} Y_{j}^{m}\left(z^{\prime}\right) d z^{\prime} d t\right) \hat{f}(\xi) Y_{j}^{m}\left(y^{\prime}\right) .
\end{aligned}
$$

But, there is a function $\phi_{m}(s), s \in \mathbf{R}$, independent of $Y \in H^{m}$ such that (see [SW, p. 152])

$$
\int_{\Sigma_{n-1}} e^{-2 \pi i t z^{\prime} \cdot \xi} Y\left(z^{\prime}\right) d z^{\prime}=\phi_{m}(s) Y\left(\xi^{\prime}\right)
$$


and we claim that

$$
\phi_{m}(s)=\frac{i^{-m}}{b_{n-2}} \frac{J_{m+(n-2) / 2}(2 \pi s)}{s^{(n-2) / 2}} .
$$

When $n \geq 3$, this can be seen by applying (1.5) with $Y\left(z^{\prime}\right)=P_{m}^{(n-2) / 2}\left(\left\langle\xi^{\prime} \cdot z^{\prime}\right\rangle\right)$ where $P_{m}^{(n-2) / 2}$ is the $m$ th Gegenbauer polynomial of order $(n-2) / 2$. Using, then, the Gegenbauer polynomial expansion of $\mathrm{e}^{-2 \pi i s z^{\prime} \cdot \xi^{\prime}}$ (see [V, p. 557]), we obtain (1.6). In the case $n=2$, apply (1.5) with $Y\left(z^{\prime}\right)=z^{\prime m}, z^{\prime} \in \Sigma_{1}$. A simple change of variable shows that the integral (1.5) equals $i^{-m} J_{m}(2 \pi s) \xi^{\prime m}$. Using, now, (1.5) and (1.6) in (*), it follows that

$$
\begin{aligned}
Q^{m}\left[F_{\varphi}(\hat{\xi}, \cdot)\right]\left(y^{\prime}\right) & =d(m, n) \sum_{j=1}^{d(m, n)} \int_{-\infty}^{+\infty} \varphi(t) \phi_{m}(t|\xi|) d t Y_{j}^{m}\left(\xi^{\prime}\right) \hat{f}(\xi) Y_{j}^{m}\left(y^{\prime}\right) \\
& =\sqrt{d(m, n)} \sum_{j=1}^{d(m, n)} i^{-m} Y_{j}^{m}\left(\xi^{\prime}\right) M_{\varphi}^{m}(\xi) \hat{f}(\xi) Y_{j}^{m}\left(y^{\prime}\right) \\
& =\sqrt{d(m, n)} \sum_{j=1}^{d(m, n)}\left(R_{j}^{m}\left(\check{M}_{\varphi}^{m} * f\right)\right)^{-}(\xi) Y_{j}^{m}\left(y^{\prime}\right) .
\end{aligned}
$$

With the help of (1.4), we then recover the computations Duoandikoetxea and Rubio de Francia made in order to obtain the boundedness on $L^{p}\left(\mathbf{R}^{\mathbf{n}}\right)$ of the higher Riesz transforms. Let us be more explicit.

We start from the formula (see [W, p. 391])

$$
\int_{0}^{+\infty} \frac{J_{\nu}(t)}{t^{\nu-\mu+1}} d t=\frac{\Gamma(\mu / 2)}{2^{\nu-\mu+1} \Gamma\left(\nu-\frac{1}{2} \mu+1\right)} .
$$

Introduce the constant

$$
\lambda_{\gamma}(m, n)=\sqrt{d(m, n)} \pi \frac{\Gamma((m-i \gamma) / 2) \Gamma(n / 2)}{\Gamma((n+m+i \gamma) / 2)},
$$

for $\gamma \in \mathbf{R}$. When $m$ is odd, apply the above formula with $\nu=(n-2) / 2+m$ and $\mu=m$. Some easy transformations give

$$
\frac{\sqrt{d(m, n)}}{b_{n-2}} \int_{-\infty}^{+\infty} \frac{1}{t} \frac{J_{m+(n-2) / 2}(2 \pi t|\xi|)}{(t|\xi|)^{(n-2) / 2}} d t=\lambda_{0}(m, n) \text {. }
$$

When $m$ is even, take $\nu=(n-2) / 2+m$ and $\mu=m-i \gamma, \gamma \neq 0$. This gives

$$
\frac{\sqrt{d(m, n)}}{b_{n-2}} \int_{-\infty}^{+\infty} \frac{1}{|t|^{1+i \gamma}} \frac{J_{m+(n-2) / 2}(2 \pi t|\xi|)}{(t|\xi|)^{(n-2) / 2}} d t=\lambda_{\gamma}(m, n)|\xi|^{i \gamma} \text {. }
$$

Let us then put $\varphi_{0}(t)=$ p.v. $\frac{1}{t}$ when $m$ is odd and $\varphi_{\gamma}(t)=1 /|t|^{1+i \gamma}$ when $m$ is even, and $\hat{f}_{\gamma}(\xi)=|\xi|^{i \gamma} \hat{f}(\xi)$ for all $\gamma$. It follows that

$$
\check{M}_{\varphi_{\gamma}}^{m} * f=\lambda_{\gamma}(m, n) f_{\gamma} .
$$

Thus, (1.4) gives us

$$
Q^{m}\left[F_{\varphi_{\gamma}}(x, \cdot)\right]\left(y^{\prime}\right)=\lambda_{\gamma}(m, n) \sqrt{d(m, n)} \sum_{j=1}^{d(m, n)} R_{j}^{m}\left(f_{\gamma}\right)(x) Y_{j}^{m}\left(y^{\prime}\right)
$$


This is a formula analogous to that obtained in [DR], but written here in terms of this projection $Q^{m}$ followed by a "transferred operator". The following estimate, due to E. Stein, can then be obtained as in [DR].

Proposition 3. For all $1<p<\infty$ and $m \geq 1$,

$$
\left\|\left(\sum_{j=1}^{d(m, n)}\left|R_{j}^{m} f\right|^{2}\right)^{1 / 2}\right\|_{L^{p}\left(\mathbf{R}^{\mathbf{n}}\right)} \leq K(m, p)\|f\|_{L^{p}\left(\mathbf{R}^{\mathbf{a}}\right)}
$$

where $K(m, p)$ is independent of $n$.

The analog to formula $(* *)$ at the beginning of this section does not hold for all $m \geq 1$. But one does have (see [DR]):

$$
\|Y\|_{L^{q}\left(\Sigma_{n-1}, d y^{\prime}\right)} \leq q^{m / 2}\|Y\|_{L^{2}\left(\Sigma_{n-1}, d y^{\prime}\right)}, \quad 1 \leq q<\infty,
$$

for all spherical harmonics of degree $m$. This is a good substitute for $(* *)$ that enables us to obtain boundedness properties of $Q^{m}$ on $L^{q}\left(\Sigma_{n-1}, d y^{\prime}\right)$, $1<q<\infty$. Let us observe again that the significance of the normalization imposed on $M_{\varphi}^{m}(\xi)$ defined in (1.1) is apparent here since it allows us to obtain the natural higher Riesz transforms.

We are now ready to prove (1.2). It is clearly enough to suppose $2 \leq$ $p<\infty$ since we are dealing with a convolution operator. From the relation $\sum_{j=1}^{d(m, n)}\left|Y_{j}^{m}\left(\xi^{\prime}\right)\right|^{2}=1$ (see [SW, p. 144]) and the definition of $R_{j}^{m}$, $j=1, \ldots, d(m, n)$, we obtain $\sum_{j=1}^{d(m, n)}\left(R_{j}^{m}\right)^{*} R_{j}^{m}=$ Id . This equality, together with (1.8) and a duality argument gives us

$$
\|f\|_{L^{p}\left(\mathbf{R}^{\mathbf{n}}\right)} \leq K\left(m, p^{\prime}\right)\left\|\left(\sum_{j=1}^{d(m, n)}\left|R_{j}^{m} f\right|^{2}\right)^{1 / 2}\right\|_{L^{p}\left(\mathbf{R}^{\mathbf{n}}\right)}, \quad 1<p<\infty,
$$

and $1 / p+1 / p^{\prime}=1$. Thus,

$$
\begin{aligned}
& \left\|\check{M}_{\varphi}^{m} * f\right\|_{L^{p}\left(\mathbf{R}^{\mathbf{n}}\right)} \leq K\left(m, p^{\prime}\right)\left\|\left(\sum_{j=1}^{d(m, n)}\left|R_{j}^{m}\left(\check{M}_{\varphi}^{m} * f\right)\right|^{2}\right)^{1 / 2}\right\|_{L^{p}\left(\mathbf{R}^{\mathbf{n}}\right)} \\
& =K\left(m, p^{\prime}\right)\|\| \sqrt{d(m, n)} \sum_{j=1}^{d(m, n)} R_{j}^{m}\left(\check{M}_{\varphi}^{m} * f\right)(x) Y_{j}\left(y^{\prime}\right)\left\|_{L^{2}\left(\Sigma_{n-1}, d y^{\prime}\right)}\right\|_{L^{p}\left(\mathbf{R}^{\mathbf{n}}\right)} \\
& \stackrel{(1.4)}{=} K\left(m, p^{\prime}\right)\|\| Q^{m}\left[F_{\varphi}(x, \cdot)\right]\left(y^{\prime}\right)\left\|_{L^{2}\left(\Sigma_{n-1}, d y^{\prime}\right)}\right\|_{L^{p}\left(\mathbf{R}^{\mathbf{n}}\right)} \\
& Q^{Q^{m} \text { projection }} \leq \mathrm{L} K\left(m, p^{\prime}\right)\|\| F_{\varphi}\left(x, y^{\prime}\right)\left\|_{L^{2}\left(\Sigma_{n-1}, d y^{\prime}\right)}\right\|_{L^{p}\left(\mathbf{R}^{\mathbf{n}}\right)} \\
& \stackrel{\text { Jensen's inequality }}{\leq} K\left(m, p^{\prime}\right)\|\| F_{\varphi}\left(x, y^{\prime}\right)\left\|_{L^{p}\left(\Sigma_{n-1}, d y^{\prime}\right)}\right\|_{L^{p}\left(\mathbf{R}^{\mathbf{n}}\right)} .
\end{aligned}
$$

Thus, using Fubini's Theorem and then (1.3), we obtain

$$
\begin{aligned}
\left\|\check{M}_{\varphi}^{m} * f\right\|_{L^{p}\left(\mathbf{R}^{\mathbf{n}}\right)} & \leq K\left(m, p^{\prime}\right)\left(\int_{\Sigma_{n-1}}\left(\int_{\mathbf{R}^{\mathbf{n}}}\left|F_{\varphi}\left(x, y^{\prime}\right)\right|^{p} d x\right) d y^{\prime}\right)^{1 / p} \\
& \leq K\left(m, p^{\prime}\right) N_{p}(\varphi)\|f\|_{L^{p}\left(\mathbf{R}^{\mathbf{n}}\right)} .
\end{aligned}
$$


Theorem 1 can be generalized using the reproducing properties of the Bessel functions. For simplicity, we denote by $M_{p}\left(\mathbf{R}^{\mathbf{n}}\right)$ the Banach algebra of multipliers $M(\xi)$ on $L^{p}\left(\mathbf{R}^{\mathbf{n}}\right)$ with norm $\|M\|_{p}=\sup \left\{\|\check{M} * f\|_{L^{p}\left(\mathbf{R}^{\mathbf{n}}\right)},\|f\|_{L^{p}\left(\mathbf{R}^{\mathbf{n}}\right)}=1\right\}$.

Theorem 4. Let $\varphi$ be a convolution kernel acting on $L^{p}(\mathbf{R})$. Then, for all $\alpha \geq 0$, $m \geq 1 \quad(1<p<+\infty)$ or $m=0 \quad(1 \leq p<+\infty$ and $\operatorname{supp} \varphi \subset[0,+\infty))$

$$
M_{\varphi}^{m, \alpha}(\xi)=\frac{\sqrt{d(m, n)}}{b_{n-2}} \frac{\Gamma\left[\frac{m+n}{2}+\alpha\right]}{\pi^{\alpha} \Gamma\left[\frac{m+n}{2}\right]} \int_{-\infty}^{+\infty} \varphi(t) \frac{J_{m+\alpha+(n-2) / 2}(2 \pi t|\xi|)}{(t|\xi|)^{\alpha+(n-2) / 2}} d t
$$

is a multiplier on $L^{p}\left(\mathbf{R}^{\mathbf{n}}\right)$ with norm less than $C(m, p) N_{p}(\varphi)(C(m, p)$ being the constant in (1.2)).

Proof. Using the reproducing formula for Bessel functions

$$
J_{\mu+\alpha}(t)=\frac{t^{\alpha}}{2^{\alpha-1} \Gamma(\alpha)} \int_{0}^{1} J_{\mu}(s t) s^{\mu+1}\left(1-s^{2}\right)^{\alpha-1} d s
$$

with $\mu=(n-2) / 2+m$ and $\alpha>0$, one finds that

$$
\begin{aligned}
M_{\varphi}^{m, \alpha}(\xi) & =\frac{\sqrt{d(m, n)}}{b_{n-2}} \int_{-\infty}^{+\infty} \varphi(t) \int_{-\infty}^{+\infty} \varphi_{m, \alpha}(s) \frac{J_{m+(n-2) / 2}(2 \pi t s|\xi|)}{(s t|\xi|)^{(n-2) / 2}} d s d t \\
& =\int_{-\infty}^{+\infty} \varphi_{m, \alpha}(s) M_{\varphi}^{m}(s \xi) d s
\end{aligned}
$$

by an application of Fubini's Theorem (justified when $\varphi$ is as in Remark 3) where

$$
\varphi_{m, \alpha}(t)=H(m, \alpha)^{-1}\left(1-s^{2}\right)^{\alpha-1} \chi_{[0,1]}(s) \in L^{1}(\mathbf{R})
$$

with $H(m, \alpha)$ such that $\left\|\varphi_{m, \alpha}\right\|_{L^{1}(\mathbf{R})}=1$. Thus, Theorem 4 follows by applying Theorem 1 and noticing that $M_{p}\left(\mathbf{R}^{\mathbf{n}}\right)$ is a Banach space in which the dilations $M(\xi) \rightarrow M(s \xi)$ are isometries.

Let us discuss whether the $L^{p}$ bounds are best possible for $M_{\varphi}^{m}$. As long as $m, p$ and $\varphi$ are fixed the dimension free estimates in Theorems 1 and 4 are best possible for large $n$. However, for any individual multiplier $M_{\varphi}^{m}$ acting on $L^{p}\left(\mathbf{R}^{\mathbf{n}}\right)$ the bound we provide need not be best possible. For example, we showed that, for $m$ odd and $\varphi(t)=$ p.v. $\frac{1}{t}, M_{\varphi}^{m}$ is constant. Thus, its multiplier norm on $L^{p}\left(\mathbf{R}^{\mathbf{n}}\right)$ is the same constant although our theorem provides the bound $K\left(m, p^{\prime}\right) N_{p}(\varphi)$ which is not even finite for $p=1$ or $p=\infty$. This is due to the fact that the integrals that define these multipliers do not converge absolutely most of the times. If the dimension $n, p$ and $\varphi$ are fixed, then $K(m, p)$ increases exponentially with $m$ (see [DR]) and is not optimal for (1.8) to hold. Indeed, another argument proving (1.8) gives a bound growing polynomially on $m$ (this bound depends, thus, on $n$ and is equal to $\sqrt{d(m, n)}\left|\lambda_{y}(m, n)\right|^{-1} C(p)$, where $C(p)=O\left(p^{2} /(p-1)\right)$ if $m$ is odd and $C(p)=O\left(p^{4} /(p-1)^{2}\right)$ if $m$ is even).

Let us consider some examples.

(1) We start from [W, p. 411]

$$
\int_{0}^{+\infty} \frac{J_{\mu}(a t) J_{\nu}(\omega t)}{w^{\nu} t^{\mu-\nu-1}} d t= \begin{cases}0 & \text { if } a<\omega, \\ \frac{\left(a^{2}-\omega^{2}\right)^{\mu-\nu-1}}{2^{\mu-\nu-1} a^{\mu} \Gamma(\mu-\nu)} & \text { if } a \geq \omega,\end{cases}
$$


valid when $\mu>\nu$. Apply this formula with $\nu=m+(n-2) / 2, \mu=m+\beta+$ $(n-2) / 2, \beta>0, a=2 \pi$ and $\omega=2 \pi|\xi|$. Let us put

$$
\psi_{m, \beta}(t)=\frac{2 \pi \Gamma(\beta)}{\pi^{\beta-1}} \frac{b_{n-2}}{\sqrt{d(m, n)}} \frac{J_{m+\beta+(n-2) / 2}(2 \pi t)}{t^{\beta-n / 2}} \chi_{[0,+\infty)}(t) .
$$

Then

$$
M_{\psi_{m, \beta}}^{m}(\xi)=|\xi|^{m}\left(1-|\xi|^{2}\right)_{+}^{\beta-1},
$$

(where $x_{+}=\max (x, 0)$ ). Here we obtain multipliers associated with BochnerRiesz means (when $m=0$ ). When $\beta-1>(n-1) / 2$ (the critical index for these means), $\psi_{m, \beta} \in L^{1}(\mathbf{R})$ for all $m \geq 0$ (see for example [SW, p. 158]) and we can apply Theorem 1 . It is also of interest to wonder whether the $L^{1}$ bound of the Bochner-Riesz means of order $\delta+(n-1) / 2, \delta>0$, is bounded independently of the dimension for fixed $\delta$. This bound is simply the $L^{1}\left(\mathbf{R}^{+}\right)$norm of $\psi_{0, \beta}$ where $\beta-1=\delta+(n-1) / 2$. So far the best estimate we can produce for this norm is $C(\delta) \sqrt{n}$.

This is obtained by writing $\int_{0}^{\infty}\left|\psi_{0, \beta}(t)\right| d t=\int_{0}^{c n} \cdots+\int_{c n}^{\infty} \cdots$ where $c$ is a small appropriately chosen number. A rough estimate obtained from the integral representation for Bessel functions [W, p. 48] implies that the integral on $[0, c n]$ is $0(1)$ as $n$ increases. On the other hand, the second integral is bounded by

$$
\left(\int_{c n}^{\infty} t^{-2 \alpha} d t\right)^{1 / 2}\left(\int_{c n}^{\infty}\left|\psi_{0, \beta}(t)\right| t^{2 \alpha} d t\right)^{1 / 2}
$$

where $\alpha$ is chosen so that $1 / 2<\alpha<\delta+1 / 2$ (recall that $\beta-1=\delta+(n-1) / 2$ ). The formula (2.4) which we state later provides, then, a bound that is $O(\sqrt{n})$ as $n$ increases for this product of integrals.

(2) Consider $\varphi(t)=\chi_{[0,1]}(t)$ and, for $r>0$, let $\varphi_{r}(t)=\varphi(t / r) / r$. Define also

$$
M_{r} f(x)=\frac{1}{\operatorname{vol}(B(0, r))} \chi_{B(0, r)} * f(x),
$$

where $B(0, r)$ is the Euclidean ball centered at 0 of radius $r$. Then

$$
M_{\varphi_{r}}^{2}(\xi)=\frac{\sqrt{d(2, n)}}{b_{n-2}} \frac{1}{r} \int_{0}^{r} \frac{J_{2+(n-2) / 2}(2 \pi t|\xi|)}{(t|\xi|)^{(n-2) / 2}} d t .
$$

Using the fact that $\frac{d}{d t}\left(J_{\mu}(t) t^{-\mu}\right)=-J_{\mu+1}(t) t^{-\mu}$, we see that

$$
M_{\varphi_{r}}^{2}(\xi)=\frac{\sqrt{d(2, n)}}{2 \pi b_{n-2}} \frac{1}{r} \int_{0}^{r} t \frac{d}{d t}\left(\frac{J_{n / 2}(2 \pi t|\xi|)}{(t|\xi|)^{n / 2}}\right) d t
$$

As is known,

$$
\left(\frac{1}{\operatorname{vol}(B(0, r))} \chi_{B(0, r)}\right)^{\hat{}}(\xi)=\frac{1}{b_{n}} \frac{J_{n / 2}(2 \pi r|\xi|)}{(r|\xi|)^{n / 2}} .
$$

A simple integration by parts gives, therefore,

$$
\check{M}_{\varphi_{r}}^{2} * f(x)=\sqrt{\frac{(n-1)(n+2)}{2 n^{2}}}\left(-M_{r} f(x)+\frac{1}{r} \int_{0}^{r} M_{t} f(x) d t\right) .
$$


Thus, in order to recover the result of E. Stein about the boundedness of the Hardy-Littlewood maximal operator on $L^{p}\left(\mathbf{R}^{\mathbf{n}}\right)$ with a norm bounded independently of $n$, we study in $\S 2$ maximal operators and $g$-functions associated to the multipliers $M_{\varphi}^{m}$.

\section{RELATED MAXIMAL OPERATORS AND $g$-FUNCTIONS}

The first natural question is to try to transfer a maximal inequality from one dimension to $n$ dimensions hoping to be able to control the norms independently of $n$. We have partially succeeded in this direction.

Theorem 5. Let $\varphi$ be a positive function on $L^{1}(\mathbf{R})$ with $\|\varphi\|_{L^{1}(\mathbf{R})}=1$. Assume that for all $1<p \leq \infty$,

$$
\left\|\sup _{r>0}\left|\varphi_{r} * g\right|\right\|_{L^{p}(\mathbf{R})} \leq A_{p}\|g\|_{L^{p}(\mathbf{R})} .
$$

Then, for $m \geq 1$ and $1<s<\min (2, p)$,

$$
\left\|\sup _{r>0}\left|\left(\check{M}_{\varphi}^{m}\right)_{r} * f\right|\right\|_{L^{p}\left(\mathbf{R}^{\mathbf{n}}\right)} \leq\left(s^{\prime}\right)^{m / 2} K(m, p) \sqrt{d(m, n)} A_{p / s}^{1 / s}\|f\|_{L^{p}\left(\mathbf{R}^{\mathbf{p}}\right)},
$$

where $K(m, p)$ is the constant in (1.8) and $1 / s^{\prime}=1-1 / s$.

Let us emphasize that to get the result for $L^{p}\left(\mathbf{R}^{\mathbf{n}}\right)$ we need the hypothesis (2.1) for $L^{p / s}(\mathbf{R})$ for some $1<s<\min (2, p)$. That is, in this case the transference is done from $L^{p / s}(\mathbf{R})$ to $L^{p}\left(\mathbf{R}^{\mathbf{n}}\right)$.

For a kernel $K$ in $\mathbf{R}^{\mathbf{n}}, K_{r}$ stands for $K(x / r) / r^{n}$. Observe that $\left(\check{M}_{\varphi}^{m}\right)_{r}=$ $\check{M}_{\varphi_{r}}^{m}$. A moment's thought shows that the case $m=0$ also holds when $\operatorname{supp} \varphi \subset$ $[0,+\infty)$ and is trivial since

$$
\left(\check{M}_{\varphi}^{0}\right)_{r} * f(x)=\int_{\Sigma_{n-1}} F_{\varphi_{r}}\left(x, y^{\prime}\right) d y^{\prime} .
$$

By using this case and $\varphi(t)=n t^{n-1} \chi_{[0,1]}(t)$, the constant $A_{p}$ in (2.1) behaves asymptotically like $n p /(p-1)$ and is sharp in $n$ as long as $p<\infty$. For the above $\varphi,\left(\check{M}_{\varphi}^{0}\right)_{r} * f(x)=M_{r} f(x)$. Thus, we do not obtain for the HardyLittlewood maximal operator (as in [SS]) the independence in $n$ of its norm by looking at $\check{M}_{\varphi_{r}}^{0}$ with that choice of $\varphi$. Notice that the above argument is that of Theorem 2 in [SS].

Proof. We prove the inequality (2.2) for $m \geq 1$. The difficulty here is to deal carefully with the Riesz transforms when using (1.4). We introduce

$$
C_{y^{\prime}} f(x)=\sup _{r>0}\left|\int_{-\infty}^{+\infty} \varphi_{r}(t) f\left(x-t y^{\prime}\right) d t\right| .
$$

By transference, it follows that for all $y^{\prime} \in \Sigma_{n-1}$

$$
\left\|C_{y^{\prime}} f\right\|_{L^{p}\left(\mathbf{R}^{\mathrm{n}}\right)} \leq A_{p}\|f\|_{L^{p}\left(\mathbf{R}^{\mathrm{n}}\right)} .
$$

Define $Q_{j}^{m}$ to be the orthogonal projection on $L^{2}\left(\Sigma_{n-1}\right)$ onto the 1-dimensional subspace generated by $Y_{j}^{m}$. From the identity $\sum_{j=1}^{d(m, n)} R_{j}^{m} R_{j}^{m}=(-1)^{m}$ Id, we 
obtain

$$
\begin{aligned}
\left|\check{M}_{\varphi_{r}}^{m} * f(x)\right| \leq & \sum_{j=1}^{d(m, n)}\left|R_{j}^{m}\left(\check{M}_{\varphi_{r}}^{m} * R_{j}^{m} f\right)(x)\right| \\
= & \sum_{j=1}^{d(m, n)} \|\left. Q_{j}^{m}\left(\int_{-\infty}^{+\infty} \varphi_{r}(t) R_{j}^{m} f(x-t \cdot) d t\right)\right|_{L^{2}\left(\Sigma_{n-1}, d y^{\prime}\right)} \\
= & \sum_{j=1}^{d(m, n)}\left|\int_{\Sigma_{n-1}} \int_{-\infty}^{+\infty} \varphi_{r}(t) R_{j}^{m} f\left(x-t z^{\prime}\right) d t \sqrt{d(m, n)} Y_{j}^{m}\left(z^{\prime}\right) d z^{\prime}\right| \\
\leq & \sum_{j=1}^{d(m, n)}\left(\int_{\Sigma_{n-1}}\left|\int_{-\infty}^{+\infty} \varphi_{r}(t) R_{j}^{m} f\left(x-t z^{\prime}\right) d t\right|^{s} d z^{\prime}\right)^{1 / s} \\
& \times\left(\int_{\Sigma_{n-1}} \sqrt{\left.d(m, n)\left|Y_{j}^{m}\left(z^{\prime}\right)\right|^{s^{\prime}} d z^{\prime}\right)^{1 / s^{\prime}}}\right.
\end{aligned}
$$

thus,

(*)

$$
\begin{aligned}
& \left|\check{M}_{\varphi_{r}}^{m} * f(x)\right| \\
& \quad \leq\left(s^{\prime}\right)^{m / 2} d(m, n)^{1 / s^{\prime}}\left(\sum_{j=1}^{d(m, n)} \int_{\Sigma_{n-1}} \int_{-\infty}^{+\infty} \varphi_{r}(t)\left|R_{j}^{m} f\right|^{s}\left(x-t z^{\prime}\right) d t d z^{\prime}\right)^{1 / s},
\end{aligned}
$$

where we have used (1.9) for the integral involving $Y_{j}^{m}$, and Jensen's inequality (since $\left\|\varphi_{r}\right\|_{L^{1}(\mathbf{R})}=1$ ) and Hölder's inequality (for the finite sum) in the other term.

Let $g(x)=\sum_{j=1}^{d(m, n)}\left|R_{j}^{m} f(x)\right|^{s}$. Observe that $g \in L^{p / s}\left(\mathbf{R}^{\mathbf{n}}\right)$ by (1.8) and

$$
g(x) \leq\left(\sum_{j=1}^{d(m, n)}\left|R_{j}^{m} f(x)\right|^{2}\right) d(m, n)^{(2-s) / 2} .
$$

Thus, $\|g\|_{L^{p / s}\left(\mathbf{R}^{\mathbf{n}}\right)} \leq d(m, n)^{(2-s) / 2} K(m, p)^{s}\|f\|_{L^{p}\left(\mathbf{R}^{\mathbf{n}}\right)}^{s}$. Now, taking supremum in $r>0$ in $(*)$, then, $p$ th-power and integrating with respect to $x$, we obtain

$$
\begin{aligned}
\| \sup _{r>0}\left|\check{M}_{\varphi_{r}}^{m} * f\right| & \|_{L^{p}\left(\mathbf{R}^{\mathbf{n}}\right)} \\
\leq & \left(s^{\prime}\right)^{m / 2} d(m, n)^{1 / s^{\prime}}\left(\int_{\mathbf{R}^{\mathbf{n}}}\left(\int_{\Sigma_{n-1}}\left|C_{y^{\prime}} g(x)\right| d y^{\prime}\right)^{p / s} d x\right)^{1 / p} \\
\leq & \left(s^{\prime}\right)^{m / 2} d(m, n)^{1 / s^{\prime}}\left(\int_{\Sigma_{n-1}}\left(\int_{\mathbf{R}^{\mathbf{n}}}\left|C_{y^{\prime}} g(x)\right|^{p / s} d x\right) d y^{\prime}\right)^{1 / p} \\
\leq & \left(s^{\prime}\right)^{m / 2} d(m, n)^{1 / s^{\prime}} A_{p / s}^{1 / s}\|g\|_{L^{p / s}\left(\mathbf{R}^{\mathbf{n}}\right)}^{1 / s}
\end{aligned}
$$

Thus, using the estimate for $\|g\|_{L^{p / s}\left(\mathbf{R}^{\mathbf{n}}\right)}$ in this inequality and observing that $1 / s^{\prime}+(2-s) / 2 s=1 / 2$, we obtain the desired estimate. 
This result, however, is not completely satisfactory. Applied to Example (2) in $\S 1$, where $\varphi(t)=\chi_{[0,1]}(t)$, we obtain

$$
\left\|\sup _{r>0}\left|-M_{r} f+\frac{1}{r} \int_{0}^{r} M_{t} f d t\right|\right\|_{L^{p}\left(\mathbf{R}^{\mathbf{n}}\right)} \leq C(n, p)\|f\|_{L^{p}\left(\mathbf{R}^{\mathbf{n}}\right)}
$$

where $C(n, p)$ behaves like $\sqrt{d(2, n)} \sim n$ as $n$ tends to $\infty$ (here, $p$ is fixed). The case $p=2$ becomes much simpler as soon as we introduce some $g$ functions.

Theorem 6. Suppose $n \geq 2$ and $m \geq 1$. Assume that $\varphi$ is supported in $[0,+\infty)$ and that

$$
B_{\varphi}=\left(\int_{0}^{\infty} t|\varphi(t)|^{2} d t\right)^{1 / 2}<+\infty
$$

then

Proof. Put

$$
\left\|\sup _{r>0}\left|\check{M}_{\varphi_{r}}^{m} * f\right|\right\|_{L^{2}\left(\mathbf{R}^{\mathbf{n}}\right)} \leq B_{\varphi} m^{-1 / 2}\|f\|_{L^{2}\left(\mathbf{R}^{\mathbf{n}}\right)}
$$

$$
\widehat{G}_{t}^{m}(\xi)=\frac{J_{m+(n-2) / 2}(2 \pi t|\xi|)}{(t|\xi|)^{(n-2) / 2}} .
$$

Rewriting (1.1) and using the Cauchy-Schwarz inequality, we have

$$
\left|\check{M}_{\varphi_{r}}^{m} * f(x)\right|=\left|\frac{\sqrt{d(m, n)}}{b_{n-2}} \int_{0}^{\infty} \frac{t}{r} \varphi\left(\frac{t}{r}\right) G_{t}^{m} * f(x) \frac{d t}{t}\right| \leq B_{\varphi} g_{m}(f)(x),
$$

where

$$
g_{m}(f)(x)=\frac{\sqrt{d(m, n)}}{b_{n-2}}\left(\int_{0}^{\infty}\left|G_{t}^{m} * f(x)\right|^{2} \frac{d t}{t}\right)^{1 / 2} .
$$

It follows that

$$
\sup _{r>0}\left|\check{M}_{\varphi_{r}}^{m} * f(x)\right| \leq B_{\varphi} g_{m}(f)(x) .
$$

Therefore, we want to prove that $\left\|g_{m}(f)\right\|_{L^{2}\left(\mathbf{R}^{\mathbf{n}}\right)} \leq m^{-1 / 2}\|f\|_{L^{2}\left(\mathbf{R}^{\mathbf{n}}\right)}$. By standard Fourier transform arguments, this is equivalent to

$$
\frac{\sqrt{d(m, n)}}{b_{n-2}}\left(\int_{0}^{\infty}\left|\widehat{G}_{t}^{m}(\xi)\right|^{2} \frac{d t}{t}\right)^{1 / 2} \leq m^{-1 / 2}
$$

for $|\xi|=1$. Since, in this case, $G_{t}^{m}(\xi) \sim t^{m}$ near 0 and $t^{-(n-1) / 2}$ near $\infty$, the above integral is bounded. In order to get its estimate we use the formula (see [W, p. 403])

$$
\begin{aligned}
& \int_{0}^{\infty} \frac{J_{\mu}(a t) J_{\nu}(a t)}{t^{\lambda}} d t \\
& \quad=\frac{(a / 2)^{\lambda-1} \Gamma(\lambda) \Gamma((\mu+\nu-\lambda+1) / 2)}{2 \Gamma((\lambda+\nu-\mu+1) / 2) \Gamma((\lambda+\mu+\nu+1) / 2) \Gamma((\lambda+\mu-\nu+1) / 2)}
\end{aligned}
$$

valid provided $\mu+\nu+1>\lambda>0$. Applying (2.4) with $a=2 \pi, \nu=\mu=$ $m+(n-2) / 2$ and $\lambda=n-1$, we get that

$$
\frac{\sqrt{d(m, n)}}{b_{n-2}}\left(\int_{0}^{\infty}\left|\widehat{G}_{t}^{m}(\xi)\right|^{2} \frac{d t}{t}\right)^{1 / 2}=\left(\frac{2 m+n-2}{(m+n-2) 2 m}\right)^{1 / 2} \leq m^{-1 / 2} .
$$


Let us see, now, the consequences of this result applied to Examples (1) and (2) in $\S 1$. Let us start with Example (1).

Consider a nonnegative real number $\delta$ and write

$$
\begin{aligned}
\left(1-|\xi|^{2}\right)_{+}^{\delta} & =\left(1-|\xi|^{2}\right)_{+}^{-(n-1) / 2}\left(1-|\xi|^{2}\right)_{+}^{\delta+(n-1) / 2} \\
& =\sum_{m=0}^{\infty} a_{m}|\xi|^{2 m}\left(1-|\xi|^{2}\right)_{+}^{\delta+(n-1) / 2}
\end{aligned}
$$

The dimension $n$ is fixed and $a_{m}=O\left(m^{-1+(n-1) / 2}\right)$ as $m$ tends to $\infty$. Let

$$
T_{*}^{\delta} f(x)=\sup _{r>0}\left|\int_{\mathbf{R}^{\mathbf{n}}} e^{2 \pi i x \cdot \xi}\left(1-|r \xi|^{2}\right)_{+}^{\delta} \hat{f}(\xi) d \xi\right|
$$

be the Bochner-Riesz mean of order $\delta$. The above equality gives us, using the notations introduced previously,

$$
T_{*}^{\delta} f(x)=T_{*}^{\delta+(n-1) / 2} f(x)+\sum_{m=1}^{\infty}\left|a_{m}\right| \sup _{r>0}\left|\left(\check{M}_{\psi_{2 m, \delta+(n+1) / 2}^{2 m}}^{2 m}\right)_{r} * f(x)\right|,
$$

where $\psi_{m, \beta}$ is defined in $\S 1$, Example 1 . We then obtain a new proof of the $L^{2}$-boundedness of $T_{*}^{\delta}, \delta>0$ (see [SW]) as follows. The first term in (2.5) is known to be bounded on $L^{p}\left(\mathbf{R}^{\mathbf{n}}\right), 1<p \leq \infty$. For $m \geq 1$, Theorem 6 gives us

$$
\left\|\sup _{r>0}\left|\left(\check{M}_{\psi_{2 m, \delta+(n+1) / 2}}^{2 m}\right)_{r} * f\right|\right\|_{L^{2}\left(\mathbf{R}^{\mathbf{n}}\right)} \leq B_{\psi_{2 m, \delta+(n+1) / 2}} m^{-1 / 2}\|f\|_{L^{2}\left(\mathbf{R}^{\mathbf{n}}\right)} .
$$

The constant " $B$ " can be estimated using (2.4). Its behavior as $m$ tends to $\infty$ is asymptotic to $C(n, \delta) m^{-\delta} d(2 m, n)^{-1 / 2}$. Since $d(k, n)=O\left(k^{n-2}\right)$ as $k \rightarrow \infty$ (see the beginning of $\S 1$ ), one finds that the constant in the right-hand side of (2.6) behaves asymptotically like $C(n, \delta) m^{-\delta-(n-1) / 2}$ as $m$ increases. Thus, we obtain in (2.5)

$$
\begin{aligned}
\left\|T_{*}^{\delta} f\right\|_{L^{2}\left(\mathbf{R}^{\mathbf{n}}\right)} & \leq\left\|T_{*}^{\delta+(n-1) / 2} f\right\|_{L^{2}\left(\mathbf{R}^{\mathbf{n}}\right)}+\left(\sum_{m=1}^{\infty} \frac{C(n, \delta)}{m^{1+\delta}}\right)\|f\|_{L^{2}\left(\mathbf{R}^{\mathbf{n}}\right)} \\
& \leq C^{\prime}(n, \delta)\|f\|_{L^{2}\left(\mathbf{R}^{\mathbf{n}}\right)} .
\end{aligned}
$$

By interpolation, the previous inequality gives us the boundedness of $T_{*}^{\delta}$ on $L^{p}\left(\mathbf{R}^{\mathbf{n}}\right)$ when $\delta>|1 / 2-1 / p|(n-1)$. This is, of course, nothing new. This argument can be extended when replacing the means $\left(1-|\xi|^{2}\right)_{+}^{\delta}$ by means that have wilder singularities near $|\xi|=1$. As an example, we get

Corollary 7. Let $\beta>1$. Then, the maximal operator associated to the multiplier $\left(1-\log \left(1-|\xi|^{2}\right)_{+}\right)^{-\beta}$ is bounded on $L^{2}\left(\mathbf{R}^{\mathbf{n}}\right)$.

This result is known by keeping track of the constants in the classical proof for the $L^{2}$-boundedness of the operator $T_{*}^{\delta}$ (we thank A. Carbery for pointing this out to us).

Proof. Write for $|\xi|<1$ and any $\delta>0$,

$$
\begin{aligned}
(1- & \left.\log \left(1-|\xi|^{2}\right)\right)^{-\beta} \\
& =\left\{\left(1-\log \left(1-|\xi|^{2}\right)\right)^{-\beta}\left(1-|\xi|^{2}\right)^{-(n-1) / 2-\delta}\right\}\left(1-|\xi|^{2}\right)^{(n-1) / 2+\delta} \\
& =\sum_{m=0}^{\infty} b_{m}|\xi|^{2 m}\left(1-|\xi|^{2}\right)^{(n-1) / 2+\delta} .
\end{aligned}
$$


We expanded here the function within curly brackets in power series. The behavior of $b_{m}$ (see [Z, Vol. I, p. 192]) is asymptotic to $m^{(n-1) / 2+\delta-1}(\log m)^{-\beta}$. Using, then, (2.6) we get that

$$
\begin{aligned}
& \left\|\sup _{R>0}\left|\int_{|\xi|<R^{-1}} \mathrm{e}^{2 \pi i x \cdot \xi}\left(1-\log \left(1-|R \xi|^{2}\right)\right)^{-\beta} \hat{f}(\xi) d \xi\right|\right\|_{L^{2}\left(\mathbf{R}^{\mathbf{n}}\right)} \\
& \quad \leq C(n, \delta, \beta) \sum_{m \geq 2}^{\infty} \frac{1}{m \log ^{\beta} m}\|f\|_{L^{2}\left(\mathbf{R}^{\mathbf{n}}\right)} .
\end{aligned}
$$

This completes the proof.

We hope that this approach can be extended to other values of $p, p \neq 2$. This would require either a control on the constant $D(m)$ such that

$$
\left\|g_{m}(f)\right\|_{L^{p}\left(\mathbf{R}^{\mathrm{n}}\right)} \leq D(m)\|f\|_{L^{p}\left(\mathbf{R}^{\mathbf{n}}\right)}
$$

for $p$ in the range $[2,2 n /(n-1)]$ or a better control of the constant in inequality (2.2). It should be noticed that the two above results show that the estimate in Theorem 6 is sharp as far as the behavior in $m$ of the bound is concerned. This can also be seen from a simple calculation showing that the $L^{2}$-bound for the multiplier $|\xi|^{m}\left(1-|\xi|^{2}\right)_{+}^{\beta-1}$ is equivalent as $m$ tends to $\infty$ to the $L^{2}$-bound given by Theorem 6 for its associated maximal operator.

At this point, let us make the following remark. We were first interested in the behavior in $n$ of the various constants occurring in (1.7), (1.8), (1.9) and (2.2). However, the price we have to pay is an exponential growth in $m$ of these constants. With regard to the above example, the behavior in $m$ turns to be essential when $n$ is fixed. Then, a reexamination of these inequalities shows that we can obtain, for $n$ fixed, constants growing polynomially in $m$. This could be useful for extending the above ideas.

We examine, now, Example (2) in $\S 1$ in order to obtain dimension free estimates for the Hardy-Littlewood operator. From Theorem 6 applied with $\varphi(t)=\chi_{[0,1]}(t)$ and formula (1.10), we get that

$$
\left\|\sup _{r>0}\left|M_{r} f-\frac{1}{r} \int_{0}^{r} M_{t} f d t\right|\right\|_{L^{2}\left(\mathbf{R}^{\mathbf{n}}\right)} \leq \sqrt{\frac{n}{n-1}}\|f\|_{L^{2}\left(\mathbf{R}^{\mathbf{n}}\right)} .
$$

A precise estimate will be obtained for the Hardy-Littlewood maximal operator $M f(x)=\sup _{r>0}\left|M_{r} f(x)\right|$ as soon as we control the error term.

Let us introduce first the Hardy-Littlewood maximal operator in a direction $y$ by

$$
M_{y} f(x)=\sup _{r>0}\left|\frac{1}{r} \int_{0}^{r} f(x-t y) d t\right|, \quad y \in \mathbf{R}^{\mathbf{n}} .
$$

Once again, by transference,

$$
\left\|M_{y} f\right\|_{L^{p}\left(\mathbf{R}^{\mathbf{n}}\right)} \leq C(p)\|f\|_{L^{p}\left(\mathbf{R}^{\mathbf{n}}\right)},
$$

where $C(p)$ is the norm of the 1-dimensional Hardy-Littlewood maximal operator. A sharp value for $C(p)$ is provided by the following result.

Lemma 8. $C(p)=p /(p-1), 1<p \leq \infty$.

This lemma is proved in $[D$, p. 233]. However, we shall present a slightly different argument which we postpone for a moment. Let us first conclude our study of the $n$-dimensional Hardy-Littlewood operator. 
Proposition 9. Let $K$ be a kernel in $L^{1}\left(\mathbf{R}^{\mathbf{n}}\right)$ (not necessarily radial). Then, for $1<p \leq \infty$,

$$
\left\|\sup _{r>0}\left|\frac{1}{r} \int_{0}^{r} K_{t} * f d t\right|\right\|_{L^{p}\left(\mathbf{R}^{\mathbf{n}}\right)} \leq C(p)\|K\|_{L^{1}\left(\mathbf{R}^{\mathbf{n}}\right)}\|f\|_{L^{p}\left(\mathbf{R}^{\mathbf{n}}\right)} .
$$

Proof. Write

$$
\frac{1}{r} \int_{0}^{r} K_{t} * f(x) d t=\int_{\mathbf{R}^{\mathbf{n}}} K(y)\left(\frac{1}{r} \int_{0}^{r} f(x-t y) d t\right) d y .
$$

Thus,

$$
\sup _{r>0}\left|\frac{1}{r} \int_{0}^{r} K_{t} * f d t\right| \leq \int_{\mathbf{R}^{\mathbf{n}}}|K(y)| M_{y} f(x) d y .
$$

Minkowski's inequality and (2.8) yield, therefore, estimate (2.9).

From (2.7), (2.9), Lemma 8 and interpolation with the trivial case $p=\infty$, we obtain

Theorem 10. For $2 \leq p \leq \infty$,

$$
\|M f\|_{L^{p}\left(\mathbf{R}^{\mathbf{n}}\right)} \leq\left(2+\sqrt{\frac{n}{n-1}}\right)^{2 / p}\|f\|_{L^{p}\left(\mathbf{R}^{\mathbf{n}}\right)} .
$$

The dimension free estimate for $M$ in the case $1<p<2$ is also known (see [SW]) but our methods are limited to the case $p \geq 2$. Note that the method in [SS] also uses some kind of transference procedure which is related to the method of rotations.

Proof of the Lemma 8. Let $f \in L^{1}(\mathbf{R}) \cap L^{\infty}(\mathbf{R})$ and, for $\lambda>0, E_{\lambda}=\{x \in$ $\left.\mathbf{R} ; M_{l} f(x)>\lambda\right\}$ where $M_{l} f(x)=\sup _{r>0} \frac{1}{r} \int_{x-r}^{x}|f(t)| d t$. It is well known from the rising sun lemma that $E_{\lambda}$ has the following structure (see [D, p. 233]): $E_{\lambda}=$ $\bigcup\left(a_{k}, b_{k}\right)$ where $\left(a_{k}, b_{k}\right)$ are disjoint intervals and $\int_{a_{k}}^{b_{k}}|f(t)| d t=\lambda\left(b_{k}-a_{k}\right)$. Therefore, $m\left(E_{\lambda}\right)=\frac{1}{\lambda} \int_{E_{\lambda}}|f(t)| d t$.

Let $1<p<\infty$. Then

$$
\begin{aligned}
\int_{\mathbf{R}} M_{l} f(x)^{p} d x & =p \int_{0}^{\infty} \lambda^{p-1} m\left(E_{\lambda}\right) d \lambda=p \int_{0}^{\infty} \lambda^{p-2} \int_{E_{\lambda}}|f(t)| d t d \lambda \\
& =p \int_{\mathbf{R}}|f(t)| \int_{0}^{M_{l} f(t)} \lambda^{p-2} d \lambda d t \quad \text { (from Fubini's Theorem) } \\
& =\frac{p}{p-1} \int_{\mathbf{R}}|f(t)|\left|M_{l} f(t)\right|^{p-1} d t .
\end{aligned}
$$

Thus, by Hölder's inequality

$$
\left\|M_{l} f\right\|_{L^{p}\left(\mathbf{R}^{\mathbf{n}}\right)} \leq \frac{p}{p-1}\|f\|_{L^{p}(\mathbf{R})} .
$$

The sharpness of the bound $p /(p-1)$ comes from the fact that for $f$ with support in $[0, \infty), M_{l} f(x)$ dominates pointwise $H f(x)=\left|\frac{1}{x} \int_{0}^{x} f(t) d t\right|$ for which the Hardy inequality

$$
\|H f\|_{L^{p}\left(\mathbf{R}^{+}\right)} \leq p /(p-1)\|f\|_{L^{p}\left(\mathbf{R}^{+}\right)}
$$

is best possible. 


\section{A GENERAL ESTIMATE FOR MAXIMAL OPERATORS ASSOCIATED TO RADIAL KERNELS}

We show here the following result.

Theorem 11. Let $K(y)$ be a radial kernel in $L^{1}\left(\mathbf{R}^{\mathbf{n}}\right)$. Then, for $n \geq 4$ and $p \geq 2$,

$$
\left\|\sup _{r>0}\left|K_{r} * f\right|\right\|_{L^{p}\left(\mathbf{R}^{\mathbf{n}}\right)} \leq\left(C(2)+\sqrt{\frac{n-2}{n-3}}\right)^{2 / p}\|K\|_{L^{1}\left(\mathbf{R}^{\mathbf{n}}\right)}\|f\|_{L^{p}\left(\mathbf{R}^{\mathbf{n}}\right)} .
$$

Before starting the proof, let us say that the interest of this result is to give a precise estimate for this maximal inequality. (3.1) strengthens the maximal inequality in Stein's book [S3, p. 63] using the least decreasing radial majorant of $K$. However, inequality (3.1) depending on $\|K\|_{L^{1}\left(\mathbf{R}^{\mathbf{n}}\right)}$ is a consequence of the boundedness of the spherical maximal operator for $n /(n-1)<p \leq \infty$ (see [S2] for the case $n \geq 3$ and [B] for the case $n=2$ ) but without giving us a precise behavior, as a function of $p$ and $n$, of the constant in (3.1).

Let us begin now the proof of (3.1).

Proof. The basic ingredient will be the following formula obtained by integration by parts (in the sense of distributions):

$$
K_{r} * f=\frac{1}{r} \int_{0}^{r} K_{s} * f d s+\frac{1}{r} \int_{0}^{r} s \frac{d}{d s} K_{s} * f d s .
$$

The first term of the right-hand side is already controlled by Proposition 9. To take care of the second term, we observe that

$$
\sup _{r>0}\left|\frac{1}{r} \int_{0}^{r} s \frac{d}{d s} K_{s} * f(x) d s\right| \leq\left(\int_{0}^{\infty}\left|s \frac{d}{d s} K_{s} * f(x)\right|^{2} \frac{d s}{s}\right)^{1 / 2} \equiv \tilde{g}(f)(x) .
$$

Thus, Theorem 11 will follow by interpolation between the case $p=2$ and $p=+\infty$, and from the following lemma (here, we suppose $\|K\|_{L^{1}\left(\mathbf{R}^{\mathbf{n}}\right)}=1$ ).

\section{Lemma 12.}

$$
\|\tilde{g}(f)\|_{L^{2}\left(\mathbf{R}^{\mathbf{n}}\right)} \leq \sqrt{\frac{n-2}{n-3}}\|f\|_{L^{2}\left(\mathbf{R}^{\mathbf{n}}\right)} .
$$

Proof. Using Plancherel's Theorem, it suffices to show that

$$
M(\xi)=\left(\int_{0}^{\infty}\left|s \frac{d}{d s} \widehat{K}_{s}(\xi)\right|^{2} \frac{d s}{s}\right)^{1 / 2} \leq \sqrt{\frac{n-2}{n-3}} .
$$

From $\S 1$, we have that

$$
\widehat{K}_{s}(\xi)=\frac{1}{b_{n-2}} \int_{0}^{\infty} \varphi(t) \frac{J_{(n-2) / 2}(2 \pi s t|\xi|)}{(s t|\xi|)^{(n-2) / 2}} d t
$$

where $\varphi(t)=\omega_{n-1} t^{n-1} K^{o}(t), t \geq 0$, and $K^{o}$ is defined by $K^{o}(|x|)=K(x)$ since $K$ is radial. Using, then, that $\frac{d}{d t}\left(J_{\mu}(t) t^{-\mu}\right)=-J_{\mu+1}(t) t^{-\mu}$, we obtain

$$
s \frac{d}{d s} \widehat{K}_{s}(\xi)=\frac{-2 \pi}{b_{n-2}} \int_{0}^{\infty} \varphi(t) \frac{J_{n / 2}(2 \pi s t|\xi|)}{(s t|\xi|)^{(n-4) / 2}} d t .
$$


By homogeneity we can suppose $|\xi|=1$. From the fact that $\int_{0}^{\infty}|\varphi(t)| d t=1$ and Jensen's inequality, we get

$$
\begin{aligned}
M(\xi) & \leq \frac{2 \pi}{b_{n-2}}\left(\int_{0}^{\infty}\left(\int_{0}^{\infty} \frac{\left(J_{n / 2}(2 \pi s t)\right)^{2}}{(s t)^{n-4}} \frac{d s}{s}\right)|\varphi(t)| d t\right)^{1 / 2} \\
& =\frac{2 \pi}{b_{n-2}}\left(\int_{0}^{\infty} \frac{\left(J_{n / 2}(2 \pi s)\right)^{2}}{(s)^{n-3}} d s\right)^{1 / 2} .
\end{aligned}
$$

We use, then equality (2.4) which is valid for $n>3$ and obtain the desired estimate for $M(\xi)$.

Some remarks. (1) Consider the kernels $K^{\alpha}(y)=\beta(\alpha)^{-1}\left(1-|y|^{2}\right)_{+}^{\alpha-1}$ for $\alpha>0$, with $\beta(\alpha)$ so that $\left\|K^{\alpha}\right\|_{L^{1}\left(\mathbf{R}^{\mathbf{n}}\right)}=1$. In this case, we have

$$
\widehat{K}^{\alpha}(\xi)=\frac{\Gamma(\alpha+n / 2)}{\pi^{\alpha-1+n / 2}} \frac{J_{\alpha+(n-2) / 2}(2 \pi|\xi|)}{|\xi|^{\alpha+(n-2) / 2}} .
$$

For this kernel, the same proof applies more easily and we get that

$$
\|\tilde{g}(f)\|_{L^{2}\left(\mathbf{R}^{\mathbf{n}}\right)}=\sqrt{\frac{n+2 \alpha-2}{n+2 \alpha-3}}\|f\|_{L^{2}\left(\mathbf{R}^{\mathbf{n}}\right)}
$$

whenever $n+2 \alpha-3>0$ and $n \geq 2$. For $\alpha=1$ and $K^{1}=\chi_{B(0,1)} / \operatorname{vol}(B(0,1))$, we find again the expression $\sqrt{n /(n-1)}$.

(2) Theorem 11 holds for radial bounded measures in $\mathbf{R}^{\mathbf{n}}$ (as well as Proposition 8 for any bounded measure in $\mathbf{R}^{\mathbf{n}}$ ). This is obtained by a simple limiting argument by approximating any radial measure $\mu$ with a sequence of $L^{1}$ radial kernels $K_{n}$ (say, in the sense of distributions) such that $\left\|K_{n}\right\|_{L^{1}\left(\mathbf{R}^{\mathbf{n}}\right)} \leq\|\mu\|_{M\left(\mathbf{R}^{\mathbf{n}}\right)}$. In particular, this gives the boundedness of the spherical maximal operator on $L^{p}\left(\mathbf{R}^{\mathbf{n}}\right)$ for $p \geq 2$ and $n \geq 4$ as in [SWa].

(3) As a consequence of the result of E. Stein on spherical maximal functions (see [S2]), an estimate of the kind

$$
\left\|\sup _{r>0}\left|K_{r} * f\right|\right\|_{L^{p}\left(\mathbf{R}^{\mathbf{n}}\right)} \leq C(p)\|K\|_{L^{1}\left(\mathbf{R}^{\mathbf{n}}\right)}\|f\|_{L^{p}\left(\mathbf{R}^{\mathbf{n}}\right)}, \quad 1<p \leq 2,
$$

is false even if $C(p)$ is allowed to depend on $n$ (consider, for example, kernels $K^{\alpha}$ with $0<\alpha \leq 1 / n$. Their related maximal operators are not bounded on $\left.L^{p}\left(\mathbf{R}^{\mathbf{n}}\right), 1<p \leq n /(n-1)\right)$.

\section{REFERENCES}

[B] J. Bourgain, Averages in the plane over convex curves and maximal operators, J. Analyse Math. 47 (1986), 69-85.

[CW] R. Coifman and G. Weiss, Transference methods in analysis, CBMS Regional Conf. Ser. in Math., no. 31, Amer. Math. Soc., Providence, R.I., 1976.

[CZ] A. P. Calderón and A. Zygmund, On higher gradients of harmonic functions, Studia Math. 24 (1964), 211-226.

[D] P. Duren, Theory of $H^{p}$ spaces, Academic Press, New York, 1970.

[DR] J. Duoandikoetxea and J. L. Rubio de Francia, Estimations indépendantes de la dimension pour les transformées de Riesz, C. R. Acad. Sci. Paris Ser. I 300 (1985), 143-162. 
[P] G. Pisier, Riesz transforms: a simpler analytic proof of P. A. Meyer's inequality, Lecture Notes in Math., vol. 1321, Springer-Verlag, 1988, pp. 485-501.

[S1] E. M. Stein, Some results in harmonic analysis in $\mathbf{R}^{\mathbf{n}}$, for $n \rightarrow \infty$, Bull. Amer. Math. Soc. (N.S.) 9 (1983).

[S2] _ Maximal functions, spherical means, Proc. Nat. Acad. Sci. U.S.A. 73 (1976), 21742175.

[S3] _ Singular integrals and differentiability properties of functions, Princeton Univ. Press, Princeton, N.J., 1970.

[SS] E. M. Stein and J. O. Strömberg, Behavior of maximal functions in $\mathbf{R}^{\mathbf{n}}$ for large $n$, Ark. Mat. 21 (1983), 259-269.

[SW] E. M. Stein and G. Weiss, Introduction to Fourier analysis on Euclidean spaces, Princeton Univ. Press, Princeton, N.J., 1971.

[SWa] E. M. Stein and S. Wainger, Problems in harmonic analysis related to curvature, Bull. Amer. Math. Soc. 84 (1978), 1239-1295.

[V] N. J. Vilenkin, Special functions and the theory of group representations, Transl. Math. Monos., vol. 22, Amer. Math. Soc., Providence, R.I., 1968.

[W] G. N. Watson, Theory of Bessel functions, 2nd ed., Cambridge Univ. Press, 1952.

[WW] J. Whittaker and G. N. Watson, Modern analysis, 4th ed., Cambridge Univ. Press, 1962.

[Z] A. Zygmund, Trigonometric series, 2nd ed., Cambridge Univ. Press, 1959.

Department of Mathematics, Washington University, St. Louis, Missouri 63130

Current address, P. Auscher: IRMAR, Université de Rennes I, Campus de Beaulieu, 35042

Rennes Cedex, France

E-mail address: auscher@roland univ-rennes.fr

Current address, M. J. Carro: Departament de Mathemàtiques, Universitat Autònoma de Barcelona, 88193 Bellaterra (Barcelona), Spain

E-mail address: carro@cerber.ub.es 